\title{
Monoacylglycerol 0-acyltransferase 1 is required for adipocyte differentiation in vitro but does not
}

\section{affect adiposity in mice}

\author{
Jason M. Singer ${ }^{1}$, Trevor M. Shew ${ }^{1}$, Daniel Ferguson ${ }^{1}$, M. Katie Renkemeyer ${ }^{1}$, Terri A. Pietka ${ }^{1}$, \\ Angela M. Hall ${ }^{1}$, Brian N. Finck ${ }^{1}$, and Andrew J. Lutkewitte ${ }^{1 *}$
}

${ }^{1}$ Center for Human Nutrition, Washington University School of Medicine, St. Louis, MO

Running title: Mogat1 regulates adipocyte differentiation

Key words: adipogenesis, monoacylglycerol, obesity, lipids

*Acceptance of proofs and correspondence to Andrew J. Lutkewitte: alutkew@wustl.edu

Center for Human Nutrition, Washington University School of Medicine, 660 Euclid Ave., Box 8031, St. Louis, MO 63110, United States.

Word Count: 4320

Funding: This work was supported by grants from the NIH R56 DK111735 and the American Diabetes

Association 1-17-IBS-109 to BNF, NIH K01 DK126990 to AJL and core laboratories of Washington

University School of Medicine: Digestive Diseases Research Cores Center NIH P30DK052574, Nutrition

Obesity Research Center P30 DK056341 and the Diabetes Research Center NIH P30 DK020579.

Disclosures: The authors have no disclosures. 


\section{STUDY IMPORTANCE}

\section{What is already known?}

- Adipose tissue expansion through adipocyte precursor cell differentiation is critical for proper lipid storage during nutrient overload.

- Monoacylglycerol O-acyltransferase 1 (Mogat1), a lipogenic enzyme, is highly induced during adipocyte differentiation of human and mouse precursor cells and is reduced in patients with obesity and metabolic dysfunction.

\section{What does this study add?}

- Mogat1 deletion during early adipocyte differentiation reduces differentiation capacity, adipogenic gene expression and lowers glycerolipid content of differentiated adipocytes.

- Adipocyte Mogat1 expression is dispensable for adiposity and metabolic outcomes high-fat fed mice and suggests compensation from other glycerolipid synthesis enzymes.

How might these results change the direction of research?

- Understanding the molecular mechanisms of glycerolipid metabolism healthy adipose tissue expansion. 


\section{ABSTRACT}

Objective: Monoacylglycerol O-acyltransferase 1 (Mogat1), a lipogenic enzyme that converts monoacylglycerol to diacylglycerol, is highly expressed in adipocytes and may regulate lipolysis by reesterifying fatty acids released during times when lipolytic rates are low. However, the role of Mogat1 in regulating adipocyte fat storage during differentiation and diet-induced obesity is relatively understudied.

Methods: Here we generated adipocyte-specific Mogat1 knockout mice and subjected them to a highfat diet to determine the effects of Mogat1 deficiency on diet-induced obesity. We also used Mogat1 floxed mice to develop preadipocyte cell lines wherein Mogat1 could be conditionally knocked out to study adipocyte differentiation in vitro.

Results: In preadipocytes, we found that Mogat1 knockout at the onset of preadipocyte differentiation prevented the accumulation of glycerolipids and reduced the differentiation capacity of preadipocytes. However, the loss of adipocyte Mogat1 did not affect weight gain or fat mass induced by high-fat diet in mice. Furthermore, loss of Mogat1 in adipocytes did not affect plasma lipid or glucose concentrations or insulin tolerance.

Conclusions: These data suggest Mogat1 may play a role in adipocyte differentiation in vitro but not adipose tissue expansion in response to nutrient overload in mice. 


\section{INTRODUCTION}

Adipose tissue regulates systemic metabolism through endocrine factors (adipokines) and the regulated storage and release of free fatty acids (FFA) and glycerol. Triglycerides comprise three fatty acids esterified to a glycerol backbone and represent the most abundant lipid species stored within adipocytes. In higher organisms, two pathways have evolved for TAG synthesis that converge at diacylglycerol (DAG) synthesis, the glycerol-3-phosphate and the monoaclylglycerol O-acyltransferase pathways. The monoacylglycerol O-acyltransferase (MGAT) family of enzymes acylate monoacylglycerol (MAG) into DAG; most of which is directly acylated into triglyceride (TAG) via diacylglycerol Oacyltransferase (DGAT) activity. In humans, three genes encode enzymes of this family (MOGAT1, MOGAT2, and MOGAT3), but there are only two Mogat family genes (Mogat1 and Mogat2) in mice. Mogat2 is enriched in intestinal enterocytes and is important for dietary fat absorption (1-3). In contrast, Mogat1 is highly induced in liver of mice with hepatic steatosis and is the primary MGAT isoform expressed in adipose tissue of humans and rodents (4-6). While intestinal MGAT function has been studied for many years, less is known about the physiologic function of MGAT in other organs. We have recently shown that hepatic and constitutive Mogat1 deletion does not affect hepatic steatosis and insulin resistance in mice in the context of diet-induced obesity (7). However, it remains to be determined whether modulating MGAT activity in adipose tissue specifically might have efficacy for preventing or treating obesity-related metabolic disease.

Mogat1 expression and MGAT activity are highly induced during differentiation of mouse and human precursors cells into mature adipocytes (4). Adipose tissue Mogat1 gene expression is reduced in genetic mouse models of marked obesity ( $d b / d b$ mice) and abdominal fat of people with obesity that exhibit metabolic abnormalities compared to people with metabolically-healthy obesity (4). Expression of Mogat1 in abdominal adipose tissue also inversely correlates to basal rates of FFA release (a measure of adipose tissue lipolysis) (4). Similarly, mice lacking Mogat1 in adipose tissue have increased basal 
lipolysis on standard chow diets (4) and Mogat1 expression and activity are reduced by fasting when lipolytic rates are high (4). Overall, these data suggest that MGAT activity serves to re-esterify FFA to limit FFA release when lipolytic rates should be low $(6,8)$ and that the loss of Mogat1 could lead to "leak" of FFAs into circulation and deposition in other tissues leading to deleterious effects such as insulin resistance in skeletal muscle and liver (9). Indeed, adipose tissue expression of MOGAT1 positively correlates to glucose disposal rates during a hyperinsulinemic-euglycemic clamp in people with obesity (4). However, this phenomenon is associative only and the cause and effect relationship between these observations requires further study.

Several questions remain regarding the role of Mogat1 in adipocyte differentiation and function. For example, although Mogat1 expression is highly induced early in the adipogenic program (4), the requirement for Mogat1 in adipocyte differentiation remains unclear. Does diminished adipose tissue MGAT activity lead to ectopic fat accumulation and metabolic abnormalities with nutrient overload? Few studies have addressed the requirement for Mogat1 in adipose tissue expansion during the progression of obesity (10-12) and those that have, used global knockout of Mogat1 rather than deleting in an adipocyte-specific manner. We have recently shown global deletion of Mogat1 actually leads to unexpected increases in weight gain in mice fed a high-fat diet (7). Herein, we sought to determine the functional consequences of Mogat1 depletion during adipocyte differentiation in vitro and expansion in vivo by using mice harboring a floxed allele of Mogat1 and by generating adipocytes with conditional deletion of Mogat1.

\section{METHODS}

\section{Animal studies.}

All mouse studies were approved by the Institutional Animal Care and Use Committee of Washington University. Mogat1 whole body null mice and adipocyte-specific Mogat1 knockout mice were generated as previously described $(4,7)$. All mice were backcrossed onto the $\mathrm{C} 57 \mathrm{BL} / 6 \mathrm{~J}$ 
background. Littermate controls were all homozygous for the Mogat1 floxed allele. Mice were group housed and given free access to food and water and subjected to a $12 \mathrm{~h}$ light/dark cycle.

Eight-week-old male and female mice were given either a control low-fat diet (LFD, Research Diets, $10 \mathrm{kcal} \%$ fat matched sucrose, D12450J) or a high-fat diet (HFD, Research Diets, $60 \mathrm{kcal} \%$ fat, D12492) for the duration indicated. Before sacrifice, mice were fasted for $4 \mathrm{~h}$ starting at 0900 and were euthanized via $\mathrm{CO}_{2}$ asphyxiation. Blood was collected from venipuncture of the inferior vena cava into EDTA-coated tubes and plasma was removed by centrifugation. Adipose and other tissues were immediately collected, flash-frozen in liquid nitrogen, and stored at $-80^{\circ} \mathrm{C}$ until further use.

\section{Metabolic phenotyping.}

Glucose tolerance tests (GTT) were performed in $5 \mathrm{~h}$ fasted mice with the fasting beginning at 0900. Glucose was dissolved in saline and mice were given intraperitoneal (IP) injections of glucose solution ( $1 \mathrm{~g} / \mathrm{kg}$ lean mass). Insulin tolerance tests (ITT) were performed in mice fasting $4 \mathrm{~h}$ starting at 0900. Insulin was given as an IP injection of recombinant Humulin $R^{\circledR}(0.75 \mathrm{U} / \mathrm{kg}$ lean mass in saline).

Blood glucose in all studies was measured from a drop of tail blood using a One Touch Ultra glucometer (Life Scan Inc.) at times indicated. Lean and fat mass were determined in fed mice by ECHO MRI.

\section{Adipocyte sizing and counting.}

Adipocyte size and number were measured as previously described $(13,14)$. Briefly, harvested inguinal and gonadal white adipose tissue $(25-50 \mathrm{mg}$ ) was fixed in $0.2 \mathrm{~mol} / \mathrm{L}$ collidine $\mathrm{HCl} / 31 \mathrm{mg} / \mathrm{mL}$ osmium tetraoxide solution for 30 days followed by dissociation with $8 \mathrm{~mol} / \mathrm{L}$ urea and $154 \mathrm{mmol} / \mathrm{L}$ $\mathrm{NaCl}$. Liberated adipocytes were measured using a 400 micrometer aperture on a Multisizer 3 (Beckman Coulter).

\section{Plasma analytes.}

Plasma lipids were determined enzymatically using commercially available kits: triglycerides (Infinity Triglyceride colorimetric assay kit (Thermo Fisher, TR22421), non-esterified free fatty acids 
(WAKO), and free glycerol reagent (Sigma, F6428).

Isolation of stromal vascular fractions.

Male or female mice were euthanized via $\mathrm{CO}_{2}$ asphyxiation and inguinal subcutaneous fat pads were isolated under sterile conditions (15). The resulting fat pads were rinsed in PBS and minced in icecold digestion buffer $(7.5 \mathrm{mg} / \mathrm{ml}$ Collagenase D (Roche 1108886001$)$, and $4.8 \mathrm{mg} / \mathrm{ml}$ Dispase II (Sigma D4693) in PBS with $10 \mathrm{mM}$ calcium). Samples were incubated in digestion buffer for $5 \mathrm{~min}$ in a $37^{\circ} \mathrm{C}$ water bath and transferred to an orbital shaker at $37^{\circ} \mathrm{C} / 5 \% \mathrm{CO}_{2}$ for $30-40 \mathrm{~min}$. Following incubation, cells were passed through a 70 micron filter and centrifuged at $700 \mathrm{xg}$ for $5 \mathrm{~min}$. The resulting stromal vascular fraction was washed in PBS before red blood cells were lysed (ACK lysing buffer, Gibco A10492). The remaining cell pellet was washed twice more with PBS before plating (DMEM F-12, 10\% FBS, 100 $\mathrm{U} / \mathrm{mL}$ PenStrep) and allowed to expand before differentiation or immortalization.

\section{Immortalization and generating of conditional knockout cells}

SVF cells were isolated as above and on the same day a confluent $10 \mathrm{~cm}$ dish of $293 \mathrm{~T}$ cells were transfected with $1.5 \mu \mathrm{g}$ psPAX2 lentiviral packaging vector (Addgene 12260), $1.5 \mu \mathrm{g}$ pMD2.G envelop plasmid (Addgene, 12259), and $3 \mu \mathrm{g}$ pBABE-neo largeT antigen cDNA (Addgene, 1780 ref (16))using lipofectamine 2000 (Invitrogen, 11668019). Three days after the SVF media was replaced with media from the transfected 293T cells that was filtered and mixed at a 1:1 ratio with standard SVF media and viral media with added polybrene (8 $\mathrm{g} / \mathrm{mL}$, Millipore Sigma TR-10030). The infected cells were selected for using Geneticin (G418 Sulfate, $0.2 \mathrm{mg} / \mathrm{mL}$ ). Conditional knockout cells were generated by infection with media from a confluent $10 \mathrm{~cm}$ dish of $293 \mathrm{~T}$ cells transfected with $3 \mu \mathrm{g}$ pCL-Eco (Addgene, 12371 ref (17)) and $3 \mu \mathrm{g}$ MSCV CreERT2 puro (Addgene, 2276 ref (18)) and polybrene (8 $\mu \mathrm{g} / \mathrm{mL}$, Millipore Sigma TR-10030). Cells were selected by puromycin $(2 \mu \mathrm{g} / \mathrm{mL})$ and maintained in DMEM F-12 (10\% FBS).

\section{Differentiation of adipocytes.}


All cells were allowed to become confluent in 12 well plates. The following day (day 0 ) cells were treated with a differentiation cocktail mix (DIXY: $1 \mu \mathrm{M}$ dexamethasone, $2 \mu \mathrm{g} / \mathrm{mL}$ insulin, $500 \mu \mathrm{M} 3-$ isobutyl-1-1methylxathine (IBMX), and $15 \mu \mathrm{M}$ troglitazone). Every 2 days thereafter, the media was replaced with maintenance media (DMEM with $2 \mu \mathrm{g} / \mathrm{mL}$ insulin) for days indicated $(5,6$, or 10 days). For knockdown studies, Cre-ERT2 recombinase activity was activated via the addition of 4 nM tamoxifen (TAM) on Day 0 or as otherwise indicated (15). All experiments were performed in triplicate and replicated in at least three independent experiments.

\section{Oil Red $O$ staining and quantification.}

Oil Red O staining was performed on days indicated in 12 well plates. Cells were rinsed in PBS and fixed with $10 \%$ formalin buffer for $60 \mathrm{~min}$ at room temperature. Fixed cells were washed in deionized water and dehydrated with $60 \%$ isopropanol for $5 \mathrm{~min}$. Oil Red O was prepared and filtered according to the manufacturer's suggestions (Sigma 00625). Neutral lipids were stained for 5 min at room temperature, and excess Oil Red O was removed and rinsed in deionized water until clear. Oil Red O was extracted by the addition of $100 \%$ isopropanol for 5 min and absorbance was read at $492 \mathrm{~nm}$.

\section{mRNA isolation and quantitative PCR.}

Total RNA was isolated from cells or frozen adipose tissue samples using Trizol ${ }^{\mathrm{TM}}$ Plus RNA Purification System (Thermo Fischer, 12183555) according to the manufacturer's protocol. 2 ug RNA was reverse transcribed into cDNA using Taqman high capacity reverse transcriptase (Life Technologies, 43038228). Quantitative PCR was performed using Power SYBR green (Applied Biosystems, 4367659) and measured on an ABI PRISM 7500 or ABI QuantStudio 3 sequence detection system (Applied Biosystems). Results were quantified using the $2^{-\Delta \Delta C t}$ method and shown as arbitrary units relative to control groups. Primer sequences are listed in Table S1.

\section{Bulk RNA Sequencing and Analysis.}


Bulk RNA sequencing and analysis were performed at the Genome Technology Access Center at the McDonnell Genome Institute of Washington University School of Medicine. Samples were prepared according to library kit manufacturer's protocol, indexed, pooled, and sequenced on an Illumina HiSeq. Basecalls and demultiplexing were performed with Illumina's bcl2fastq software and a custom python demultiplexing program with a maximum of one mismatch in the indexing read. RNA-seq reads were then aligned to the Ensembl release 76 primary assembly with STAR version 2.5.1a (19). Gene counts were derived from the number of uniquely aligned unambiguous reads by Subread:featureCount version 1.4.6-p5 (20). Isoform expression of known Ensembl transcripts were estimated with Salmon version 0.8 .2 (21). Sequencing performance was assessed for the total number of aligned reads, total number of uniquely aligned reads, and features detected. The ribosomal fraction, known junction saturation, and read distribution over known gene models were quantified with RSeQC version $2.6 .2(22)$.

All gene counts were then imported into the R/Bioconductor package EdgeR (23) and TMM normalization size factors were calculated to adjust for samples for differences in library size. Ribosomal genes and genes not expressed in the smallest group size minus one samples greater than one count per million were excluded from further analysis. The TMM size factors and the matrix of counts were then imported into the R/Bioconductor package Limma (24). Weighted likelihoods based on the observed mean-variance relationship of every gene and sample were then calculated for all samples with the voomWithQualityWeights (25). The performance of all genes was assessed with plots of the residual standard deviation of every gene to their average log-count with a robustly fitted trend line of the residuals. Differential expression analysis was then performed to analyze for differences between conditions and the results were filtered for only those genes with Benjamini-Hochberg false-discovery rate adjusted p-values less than or equal to 0.05 .

The heatmap was generated using iDEP 9.0 (26). For each contrast extracted with Limma, global perturbations in known Gene Ontology (GO) terms, MSigDb, and KEGG pathways were detected using the R/Bioconductor package GAGE (27) to test for changes in expression of the reported log 2 fold-changes reported 
by Limma in each term versus the background log 2 fold-changes of all genes found outside the respective term. Perturbed KEGG pathways where the observed log 2 fold-changes of genes within the term were significantly perturbed in a single-direction versus background or in any direction compared to other genes within a given term with p-values less than or equal to 0.05 were rendered as annotated KEGG graphs with the R/Bioconductor package Pathview (28).

\section{Lipidomic analysis.}

Lipidomic analysis was performed at the Washington University Metabolomics Facility. Immortalized SVF cells $\left(5 \times 10^{6}\right)$ were grown in $10 \mathrm{~cm}$ dishes and treated with vehicle or DIXY for 10 days. Following differentiation, cells were pelleted in $500 \mathrm{uL} \mathrm{PBS}$, frozen in liquid nitrogen, and stored at $-80^{\circ} \mathrm{C}$ until analysis. Lipids were extracted in the presence of their internal standards. MAG, DAG, and TAG species were extracted using the Blyth and Dryer method and PA was extracted using protein precipitation before LC-MS/MS analysis. Peak area ratios of each analyte were determined from the internal standards. Quality control (QC) samples were prepared by pooling an aliquot of each study sample and ejected every four study samples. Only species with CV \%, 15\% for the QC injections were reported. Data are reported as fold change from undifferentiated vehicle-treated cells.

\section{Statistical analysis.}

Data were analyzed using GraphPad Prism software. Independent and paired T-tests, one-way analysis of variance (ANOVA), or factorial ANOVAs were performed where appropriate. Secondary posthoc analysis found differences in groups using either Tukey or Sidak's multiple comparisons were appropriate. $p<0.05$ was considered significant.

\section{RESULTS}

\section{Mogat1 is required for adipocyte differentiation in vitro.}

We have previously shown Mogat1 expression and activity are highly induced during differentiation of mouse and human adipocytes in vitro (4), but whether this is critical to the differentiation process is unclear. To this end, we isolated stromal vascular fractions (SVF) from inguinal 
white subcutaneous adipose tissue (iWAT) and differentiated them using a standard differentiation cocktail (DIXY: $1 \mu \mathrm{M}$ dexamethasone, $2 \mu \mathrm{g} / \mathrm{mL}$ insulin, $500 \mu \mathrm{M}$ IBMX, $15 \mu \mathrm{M}$ troglitazone) (Figure S1A and refs 4, 15). SVFs from wild-type mice had typical increases in adipogenic gene expression that reached maximal levels of expression around day six-post DIXY treatment (Figure S1B). Mogat1 expression was induced nearly 100-fold compared to undifferentiated cells, while Mogat2 expression actually decreased during differentiation (Figure S1C and ref (29)). We found that SVF cells from constitutive Mogat1 null mice differentiated normally (Figure S2), which is consistent with our previous studies indicating that germline deletion of Mogat1 does not alter adiposity in mice (7).

To avoid potential compensation of other enzymes with MGAT activity, which are induced during differentiation (Figure S1C), we utilized SVF cells isolated from Mogat1 floxed mice to generate stable cell lines with an inducible Cre-ERT2 recombinase system (15). Following isolation, the cells were immortalized using SV40 T-antigen and then transfected with an inducible Cre-ERT2 (Figure 1A). After reaching $100 \%$ confluency (day -1 ), the following day (day 0), cells were treated with TAM (or vehicle) and medium containing DIXY (Figure 1A). Vehicle-treated cells differentiated as expected as indicated by increased Oil Red O staining of neutral lipids (Figure 1B). However, Mogat1 knockout cells had decreased Oil Red O accumulation (Figure 1B). Importantly, TAM exposure alone is not sufficient to suppress differentiation in Mogat1 floxed or wild-type SVF cells (Figure S3). As expected, Mogat1 was highly induced by differentiation in control cells, but TAM treatment reduced differentiation-induced Mogat1 gene expression without affecting Mogat2 (Figure 1C). Given the diminished capacity to accumulate lipids with Mogat1 knockout, we also analyzed the expression of several key regulators of adipocyte differentiation (Figure 2). Expression of Adipoq, Pparg1, Pparg2, Srebf1, and Fasn were all highly induced during differentiation in control cells (Figure 2A-E). However, the activation of these genes was markedly blunted in the Mogat1 knockout cells (Figure 2A-E). These data suggest that 
Mogat1 expression is indispensable for initiation of adipocyte differentiation of SVF-derived preadipocytes in vitro.

\section{Mogat1 deletion prevents glycerolipid accumulation in differentiated adipocytes.}

Next, we performed lipidomic analyses to determine the effects of differentiation and Mogat1 knockout on glycerolipid intermediate abundance (Figure 3A). We found that several MAG species were actually decreased following differentiation and this was unaffected by Mogat1 knockout (Figure 3B). Interestingly, in wild-type cells that were differentiated, the abundance of MAG(20:4) and MAG(22:6) were extremely low compared to undifferentiated cells (Figure 3B). However, these MAG species were significantly higher in Mogat1 knockout adipocytes compared to wild-type adipocytes. The product of MGAT activity, DAGs, were increased in differentiated cells compared to undifferentiated cells and many highly abundant DAGs were reduced by Mogat1 knockout including saturated and monounsaturated DAG(16:0/X) and DAG(16:1/X) species (Figure 3C and Figure S4). Several long-chain highly unsaturated DAGs were decreased during differentiation independent of Mogat1 expression (Figure S4A, B). TAG species followed a similar trend as DAGs (Figure 3D and Figure S4-C). The DAG precursor and lipin enzymatic substrate, phosphatidic acid (PA), was mostly unchanged by differentiation (Figure 3E), but $\mathrm{PA}(34: 1)$ and PA(34:2) were decreased in differentiated Mogat1 knockout cells compared to control cells. Finally, we analyzed the expression of genes that encode glycerolipid metabolic enzymes in these cells. The expression of monoacylglycerol lipase (MgII) and both Dgat1 and Dgat2 was increased by differentiation and this increase was attenuated by Mogat1 knockout (Figure 4B, C). Interestingly, in these cells, expression of Lpin2 but not Lpin1 increased during differentiation, but was not affected by loss of Mogat1, while Lpin3 expression was increased by Mogat1 knockout in differentiated cells (Figure 4D). These data suggest that Mogat1 is required for preadipocytes to fully differentiate and store glycerolipids in vitro. 


\section{Adipocyte-specific Mogat1 knockout mice gain similar fat mass as littermate controls on a high-fat}

diet.

We have previously reported that, compared to wild-type littermate controls, mice with adipocyte-specific Mogat1 knockout (Adn-Mogat1-/- mice) have reduced fat mass on a chow diet, while whole-body Mogat1 null mice have increased body weight on HFD $(4,7)$. To further evaluate the functional role of Mogat1 in adipose tissue expansion we challenged male and female Adn-Mogat1-/mice and their floxed littermate controls with a HFD (60\% kcal from fat) or LFD ( $10 \%$ kcal from fat, matched sucrose) for 16 weeks (Figure 5 and Figure S5). Male Adn-Mogat1-/- mice fed HFD gained similar body weight and fat mass on the HFD compared to littermate controls on HFD (Figure 5A, B). Individual fat pad mass was also unaffected by genotype (Figure 5C-E). Similar results were found in female mice (Figure S5A-C). WAT samples were fixed and dissociated, and subsequently liberated adipocyte sizes were measured by a particle counter. The volume and diameter of individual iWAT adipocytes were increased by the HFD in wild-type mice but HFD did not affect adipocyte size significantly in the Adn-Mogat1-/- mice (Figure 5F). The number of iWAT adipocytes per mg tissue was decreased in HFD wild-types littermate controls (Figure 5F). There were no differences in cell volume, diameter, or number per mg tissue of gWAT between groups (Figure 5G). These data indicate adipocyte Mogat1 expression does not affect the adiposity or adipocyte size and number in mice fed a HFD.

\section{Adipocyte-specific Mogat1 knockout does not improve glucose or insulin tolerance.}

We have previously shown that Mogat1 regulates basal lipolysis in adipose explants and 3T3-L1 cells suggesting that loss of Mogat1 in adipocytes could lead to increased plasma lipids (4). However, plasma TAG, FFA, and glycerol were not different between genotypes on either diet (Figure 6A-C).

We performed glucose and insulin tolerance tests in adipocyte-specific Mogat1 knockout mice and littermate controls. HFD feeding lead to glucose and insulin intolerance, but there was no difference in tolerance between genotypes on either diet (Figure 6D, E). Likewise, plasma insulin concentrations 
were increased by HFD, but similar between groups (Figure 6F). Analogous results were observed in female mice fed a HFD (Figure S5D, E). Liver weight did not change but HFD increased hepatic TAG content in both genotypes (Figure 6G, H). These data indicate adipocyte-specific Mogat1 knockout mice have similar adiposity in response to a HFD as the littermate controls and adipocyte Mogat1 expression does not affect systemic lipid or glucose metabolism.

Transcriptomic analysis of inguinal white adipose tissue from adipocyte-specific Mogat1 knockout mice reveals few differences between genotypes.

We first assessed the expression of several lipogenic enzymes using RNA from iWAT from mice on either low fat or HFD. We did not detect any significant increase in the expression of DAG or TAG synthesizing genes (Mogat2, Dgat1, Dgat2, and Lpin1) (Figure 7A) that might compensate for the lack of Mogat1 between genotypes (Figure 7B and refs $(1,6,7,30,31)$. However, Mogat2 was increased nearly forty-fold by high-fat diet in both genotypes. Expression of classic markers of adipocyte lipogenesis were also unaffected by Mogat1 deletion except for fatty acid synthase (Fasn) which was increased in LFD-fed Mogat1 knockout mice compared to littermate controls. (Figure 7C).

To better understand the molecular pathways affected by adipocyte-specific Mogat1 deletion, we performed bulk RNA sequencing on iWAT from Adn-Mogat1-/- mice and their littermate controls after 16 weeks on HFD. This analysis revealed very few differences between genotypes (Figure S6). In fact, only Mogat1 was significantly decreased and 5 genes significantly increased given our criteria (adjusted $p$-value $<0.05, \log _{2} \mathrm{FC}<2.0$, Figure $\mathrm{S} 6 \mathrm{~B}, \mathrm{C}$ ). Pathway analysis identified several significantly downregulated GO biological process pathways including those related to fatty acid metabolism and oxidation (Figure S7A, B). While KEGG pathways suggest alterations in metabolic pathways, insulin signaling, and oxidative phosphorylation among others (Figure S7C, D). Although these pathways do not translate to any physiological differences seen in these mice (Figures 5, S5, and 6). 


\section{DISCUSSION}

Esterification and storage of free fatty acids as neutral TAG in adipocytes is vital for preserving systemic metabolic health. Multiple enzymatic pathways have evolved to synthesize TAG including the MGAT pathway (Figure 4A). MGAT activity and function have been extensively characterized in intestine $(2,8,30,32)$ and liver $(5,7,11,12,31,33-35)$. However, few studies have evaluated MGAT function in adipocytes. Here we demonstrate that Mogat1 is dispensable for adipose tissue expansion in high-fatfed mice. We also confirmed our previous report that Mogat1 is the predominant MGAT isoform enriched during in vitro differentiation of mouse preadipocytes (Figure S1 and ref. (4)). Using preadipocytes harboring the conditional Mogat1 allele, we generated knockout adipocyte cell lines and found that Mogat1 expression is vital for their differentiation into adipocytes. Mogat1 knockout reduced the accumulation of neutral lipids and the expression of pro-adipogenic genes like Pparg1, Pparg2, Srebf1, and Fasn. Lastly, Mogat1 ablation prevented the accumulation of lipids including a including the most abundant DAG and TAG species during differentiated mouse adipocytes.

We previously demonstrated that Mogat1 is dispensable for hepatic MGAT activity and steatosis development in obese mice (7). These data suggested compensation by alternative enzymes with MGAT activity and/or alternative sources for cellular TAG. In the present study, the loss of Mogat1 in adipocytes does not alter adiposity (i.e. TAG accumulation) in diet-induced obese mice. This may not be surprising given the functional redundancy of the lipid synthetic pathways. Lessons from in vitro studies imply that the acyltransferases, DGAT1 and DGAT2, are functionally redundant and compensate for the loss of either isoform in genetic deletion studies. The knockout of both DGAT isoforms is required to inhibit in vitro adipocyte differentiation (36). In adipose tissue specifically, DGAT1 completely compensates in adipose-specific DGAT2 knockout mice, while DGAT1 knockout mice are modestly leaner than their wild-type counterparts $(37,38)$. Furthermore, in the present study we found that the adipose tissue expression of Mogat2, which is normally very low, is markedly increased by HFD feeding 
in mice (Figure 7B). This is important given that MGAT2 has a higher specific activity than MGAT1 (1). It is possible that the activity of MGAT2 and other enzymes capable of mediating TAG synthesis overcomes any effect of Mogat1 deficiency.

Constitutive Mogat1 deletion does not alter adipocyte differentiation both in vivo and in vitro, yet acute loss at the onset of differentiation in vitro almost completely prevents glycerolipid accumulation and activation of the differentiation program. In addition, the adiponectin-Cre-driven deletion, which occurs after differentiation has commenced, does not affect differentiation in vivo. This could suggest Mogat1 plays a critical role at a very specific early time in the differentiation program and that chronic loss of Mogat1 results in compensatory effects. Although we have not identified a specific enzyme with compensatory activity, lipin family proteins also generate DAG. Although MGATs and lipins converge on DAG synthesis (the penultimate step in TAG synthesis) the physiological consequences of inhibiting these pathways are dissimilar in vitro and in vivo (2-4, 39-41). In contrast to prior work in other adipogenic cells $(29,42-44)$, Lpin1 and Lpin3 expression is only modestly increased during our differentiation of the SVF-derived cell lines (Figure 4D). Genetic loss of Lpin1 leads to dramatic impairments in adipogenesis both in vitro and in vivo $(45,41,43,42)$. This suggests the possibility that the failure of this particular in vitro model to increase Lpin1 expression during differentiation might explain the dramatic reliance on Mogat1 in this context and the differences with the in vivo phenotype.

Our lipidomics analysis revealed that the most abundant DAG and TAG species accumulate with differentiation and are reduced by Mogat1 knockout (Figure 3 and Figure S3). In contrast, many MAG and PA species were reduced during differentiation suggesting that these precursors are consumed to drive DAG and TAG synthesis. Lipidomic analysis has increased the breadth of knowledge regarding the importance of specific fatty acyl chains of glycero/phospholipids in metabolic regulation. Specific to this study, both arachidonic acid (20:4) and docosahexaenoic acid (22:6) have been shown to inhibit preadipocyte differentiation in vitro (46-48). In the present studies, MAG(20:4) and MAG(22:6) were 
abundant in undifferentiated cells, but not in differentiated wild-type adipocytes. The reduction that occurred during differentiation was attenuated by Mogat1 ablation. Yen and colleagues have reported that MGAT1 activity is higher for long-chain highly unsaturated MAGs, specifically MAG(20:4) (1).

Whether these MAG species can directly inhibit adipocyte differentiation remains to be explored. We acknowledge these studies are limited as our lipidomics data represent static lipid abundance and not flux into glycerolipid synthesis. Thus, these differences may be indicative of impaired differentiation and not MGAT1 enzymatic function per se.

\section{CONCLUSION}

Here we have provided evidence that Mogat1 expression initiates adipocyte differentiation in mouse iWAT preadipocytes. Furthermore, Mogat1 knockout reduces adipogenic gene expression and glycerolipid accumulation in differentiated cells. HFD-fed adipocyte-specific Mogat1 knockout mice have no discernable metabolic phenotype compared to littermate controls. These data suggest that there are compensatory mechanisms for loss of Mogat1 and/or that adipocyte Mogat1 expression is dispensable for diet-induced obesity in mice.

\section{ACKNOWLEDGMENTS}

We thank the Genome Technology Access Center in the Department of Genetics at Washington University School of Medicine for help with genomic analysis. The Center is partially supported by $\mathrm{NCl}$ Cancer Center Support Grant \#P30 CA91842 to the Siteman Cancer Center and by ICTS/CTSA Grant\# UL1 TR000448 from the National Center for Research Resources (NCRR), a component of the National Institutes of Health (NIH), and NIH Roadmap for Medical Research. Mass spectrometry was performed in the Metabolomics Facility at Washington University (P30 DK020579). This publication is solely the responsibility of the authors and does not necessarily represent the official view of NCRR or NIH. We would like to acknowledge the following investigators for their plasmid contributions to Addgene that were used in this research: Didier Trono, Bob Weinberg, Inder Verma, and Tyler Jacks. 


\section{REFERENCES}

1. Yen C-LE, Farese RV. MGAT2, a Monoacylglycerol Acyltransferase Expressed in the Small Intestine. J Biol Chem 2003;278:18532-18537.

2. Banh T, Nelson DW, Gao Y, Huang T-N, Yen M-I, Yen C-LE. Adult-onset deficiency of acyl CoA:monoacylglycerol acyltransferase 2 protects mice from diet-induced obesity and glucose intolerance. J Lipid Res 2015;56:379-389.

3. Gao Y, Nelson DW, Banh T, Yen M-I, Yen C-LE. Intestine-specific expression of MOGAT2 partially restores metabolic efficiency in Mogat2-deficient mice. J Lipid Res 2013:jIr.M035493.

4. Liss KHH, Lutkewitte AJ, Pietka T, et al. Metabolic importance of adipose tissue monoacylglycerol acyltransferase 1 in mice and humans. J Lipid Res 2018:jIr.M084947.

5. Hall AM, Kou K, Chen Z, et al. Evidence for regulated monoacylglycerol acyltransferase expression and activity in human liver. J Lipid Res 2012;53:990-999.

6. Yen C-LE, Stone SJ, Cases S, Zhou P, Farese RV. Identification of a gene encoding MGAT1, a monoacylglycerol acyltransferase. Proc Natl Acad Sci USA 2002;99:8512-8517.

7. Lutkewitte AJ, Singer JM, Shew TM, et al. Multiple antisense oligonucleotides targeted against monoacylglycerol acyltransferase 1 (Mogat1) improve glucose metabolism independently of Mogat1. Mol Metab 2021;49.

8. Cao J, Lockwood J, Burn P, Shi Y. Cloning and Functional Characterization of a Mouse Intestinal AcylCoA:Monoacylglycerol Acyltransferase, MGAT2. J Biol Chem 2003;278:13860-13866.

9. Fabbrini $\mathrm{E}$, Yoshino J, Yoshino $\mathrm{M}$, et al. Metabolically normal obese people are protected from adverse effects following weight gain. J Clin Invest 2015;125:787-795.

10. Agarwal AK, Tunison K, Dalal JS, et al. Mogat1 deletion does not ameliorate hepatic steatosis in lipodystrophic (Agpat2-/-) or obese (ob/ob) mice. J Lipid Res 2016;57:616-630.

11. Soufi N, Hall AM, Chen Z, et al. Inhibiting Monoacylglycerol Acyltransferase 1 Ameliorates Hepatic Metabolic Abnormalities but Not Inflammation and Injury in Mice. J Biol Chem 2014;289:30177-30188.

12. Hall AM, Soufi N, Chambers KT, et al. Abrogating Monoacylglycerol Acyltransferase Activity in Liver Improves Glucose Tolerance and Hepatic Insulin Signaling in Obese Mice. Diabetes 2014;63:2284-2296.

13. Porter LC, Franczyk MP, Pietka T, et al. NAD+-dependent deacetylase SIRT3 in adipocytes is dispensable for maintaining normal adipose tissue mitochondrial function and whole body metabolism. Am J Physiol Endocrinol Metab 2018;315:E520-E530.

14. Craft CS, Pietka TA, Schappe T, et al. The extracellular matrix protein MAGP1 supports thermogenesis and protects against obesity and diabetes through regulation of TGF- $\beta$. Diabetes 2014;63:1920-1932.

15. Lodhi IJ, Dean JM, He A, et al. PexRAP Inhibits PRDM16-Mediated Thermogenic Gene Expression. Cell Reports 2017;20:2766-2774. 
16. Hahn WC, Dessain SK, Brooks MW, et al. Enumeration of the Simian Virus 40 Early Region Elements Necessary for Human Cell Transformation. Molecular and Cellular Biology 2002.

17. Naviaux RK, Costanzi E, Haas M, Verma IM. The $\mathrm{pCL}$ vector system: rapid production of helper-free, hightiter, recombinant retroviruses. J Virol 1996;70:5701-5705.

18. Kumar MS, Pester RE, Chen $\mathrm{CY}$, et al. Dicer1 functions as a haploinsufficient tumor suppressor. Genes Dev 2009;23:2700-2704.

19. Dobin A, Davis CA, Schlesinger F, et al. STAR: ultrafast universal RNA-seq aligner. Bioinformatics 2013;29:15-21.

20. Liao Y, Smyth GK, Shi W. featureCounts: an efficient general purpose program for assigning sequence reads to genomic features. Bioinformatics 2014;30:923-930.

21. Patro R, Duggal G, Love MI, Irizarry RA, Kingsford C. Salmon provides fast and bias-aware quantification of transcript expression. Nature Methods 2017;14:417-419.

22. Wang L, Wang S, Li W. RSeQC: quality control of RNA-seq experiments. Bioinformatics 2012;28:21842185.

23. Robinson MD, McCarthy DJ, Smyth GK. edgeR: a Bioconductor package for differential expression analysis of digital gene expression data. Bioinformatics 2010;26:139-140.

24. Ritchie ME, Phipson B, Wu D, et al. limma powers differential expression analyses for RNA-sequencing and microarray studies. Nucleic Acids Res 2015;43:e47-e47.

25. Liu R, Holik AZ, Su S, et al. Why weight? Modelling sample and observational level variability improves power in RNA-seq analyses. Nucleic Acids Res 2015;43:e97.

26. Ge SX, Son EW, Yao R. iDEP: an integrated web application for differential expression and pathway analysis of RNA-Seq data. BMC Bioinformatics 2018;19:534.

27. Luo W, Friedman MS, Shedden K, Hankenson KD, Woolf PJ. GAGE: generally applicable gene set enrichment for pathway analysis. BMC Bioinformatics 2009;10:161.

28. Luo W, Brouwer C. Pathview: an R/Bioconductor package for pathway-based data integration and visualization. Bioinformatics 2013;29:1830-1831.

29. Sembongi H, Miranda M, Han G-S, et al. Distinct Roles of the Phosphatidate Phosphatases Lipin 1 and 2 during Adipogenesis and Lipid Droplet Biogenesis in 3T3-L1 Cells. J Biol Chem 2013;288:34502-34513.

30. Chon S-H, Zhou YX, Dixon JL, Storch J. Intestinal monoacylglycerol metabolism: developmental and nutritional regulation of monoacylglycerol lipase and monoacylglycerol acyltransferase. J Biol Chem 2007;282:33346-33357.

31. Qi J, Lang W, Connelly MA, et al. Metabolic tracing of monoacylglycerol acyltransferase-2 activity in vitro and in vivo. Analytical Biochemistry 2017;524:68-75. 
32. Cao J, Burn P, Shi Y. Properties of the mouse intestinal acyl-CoA:monoacylglycerol acyltransferase, MGAT2. J Biol Chem 2003;278:25657-25663.

33. Liss KHH, Ek SE, Lutkewitte AJ, et al. Monoacylglycerol acyltransferase 1 knockdown exacerbates hepatic ischemia-reperfusion injury in mice with hepatic steatosis. Liver Transplantation n/a.

34. Lutkewitte AJ, McCommis KS, Schweitzer GG, et al. Hepatic Monoacylglycerol Acyltransferase 1 is Induced by Prolonged Food Deprivation to Modulate the Hepatic Fasting Response. J Lipid Res 2019:jIr.M089722.

35. Hayashi Y, Suemitsu E, Kajimoto K, et al. Hepatic Monoacylglycerol O-acyltransferase 1 as a Promising Therapeutic Target for Steatosis, Obesity, and Type 2 Diabetes. Molecular Therapy - Nucleic Acids 2014;3.

36. Harris CA, Haas JT, Streeper RS, et al. DGAT enzymes are required for triacylglycerol synthesis and lipid droplets in adipocytes. J Lipid Res 2011;52:657-667.

37. Chitraju C, Walther TC, Farese RV. The triglyceride synthesis enzymes DGAT1 and DGAT2 have distinct and overlapping functions in adipocytes. J Lipid Res 2019;60:1112-1120.

38. Chitraju C, Mejhert N, Haas JT, et al. Triglyceride Synthesis by DGAT1 Protects Adipocytes from LipidInduced ER Stress during Lipolysis. Cell Metab 2017;26:407-418.e3.

39. Phan J, Reue K. Lipin, a lipodystrophy and obesity gene. Cell Metabolism 2005;1:73-83.

40. Csaki LS, Dwyer JR, Li X, et al. Lipin-1 and lipin-3 together determine adiposity in vivo. Molecular Metabolism 2014;3:145-154.

41. Mitra MS, Chen $\mathrm{Z}$, Ren $\mathrm{H}$, et al. Mice with an adipocyte-specific lipin 1 separation-of-function allele reveal unexpected roles for phosphatidic acid in metabolic regulation. PNAS 2013;110:642-647.

42. Nadra K, Médard J-J, Mul JD, et al. Cell Autonomous Lipin 1 Function Is Essential for Development and Maintenance of White and Brown Adipose Tissue. Molecular and Cellular Biology 2012;32:4794-4810.

43. Phan J, Péterfy M, Reue K. Lipin Expression Preceding Peroxisome Proliferator-activated Receptor- $\gamma$ Is Critical for Adipogenesis in Vivo and in Vitro. J Biol Chem 2004;279:29558-29564.

44. Koh Y-K, Lee M-Y, Kim J-W, et al. Lipin1 Is a Key Factor for the Maturation and Maintenance of Adipocytes in the Regulatory Network with CCAAT/Enhancer-binding Protein $\alpha$ and Peroxisome Proliferator-activated Receptor Y2. J Biol Chem 2008;283:34896-34906.

45. Péterfy M, Phan J, Xu P, Reue K. Lipodystrophy in the fld mouse results from mutation of a new gene encoding a nuclear protein, lipin. Nature Genetics 2001;27:121.

46. Petersen RK, JØrgensen C, Rustan AC, et al. Arachidonic acid-dependent inhibition of adipocyte differentiation requires PKA activity and is associated with sustained expression of cyclooxygenases. Journal of Lipid Research 2003;44:2320-2330. 
bioRxiv preprint doi: https://doi.org/10.1101/2022 02 14.480414; this version posted February 15,2022 . The copyright holder for this preprint

(which was not certified by peer review) is the author/funder, who has granted bioRxiv a license to display the preprint in perpetuity. It is made available under aCC-BY-NC-ND 4.0 International license.

47. Haugen F, Zahid N, Dalen KT, Hollung K, Nebb HI, Drevon CA. Resistin expression in 3T3-L1 adipocytes is reduced by arachidonic acid. J Lipid Res 2005;46:143-153.

48. Kim H-K, Della-Fera M, Lin J, Baile CA. Docosahexaenoic Acid Inhibits Adipocyte Differentiation and Induces Apoptosis in 3T3-L1 Preadipocytes. J Nutr 2006;136:2965-2969. 
Figure 1: Mogat1 expression is required for adipocyte differentiation initiation in vitro. Stromal vascular fractions (SVF) were isolated from iWAT adipose tissue of Mogat1 fl/fl mice. Cells were immortalized with SV40 T antigen prior to transfection with an inducible CreERT2. A: Diagram of treatment protocol for the generation of SVF and inducible Mogat1 cell lines as describe in the methods B, C: Cre recombinase activity to delete Mogat1 was activated with the addition $4 \mathrm{nM}$ tamoxifen on Day 0 while other cells were treated with vehicle. B: After 10 days of differentiation, lipid accumulation was reduced by Mogat1 knockout as shown by a representative ORO staining and quantification by extraction and measuring absorption at $492 \mathrm{~nm}$. C: Gene expression analysis as measured by qPCR. Mogat1 gene expression is induced by differentiation and inhibited by TAM while Mogat2 was unaffected by differentiation or TAM treatment. Data are expressed as mean \pm S.E.M. ${ }^{*} p<0.05$, comparisons are indicated; $n=3-4$ biological replicates. 
Figure 2: Mogat1 is required for adipogenic gene expression in vitro. Confluent cells were treated with tamoxifen ( $4 \mathrm{nM}$ ) at the onset of differentiation (via DIXY cocktail) and harvested after 10 days. A-E: Gene expression analysis as measured by qPCR: Peroxisome proliferator activated receptor gamma (Pparg) 1 and 2, Sterol regulatory element binding transcription factor 1 (Srebf1), Fatty acid synthase

(Fasn), and Adiponectin (Adipoq) were increased during differentiation and reduced by Mogat1

knockout. Data are expressed as mean \pm S.E.M. ${ }^{*} p<0.05$ vs. vehicle, $+p<0.05$ vs. control undifferentiated cells; $n=4$ biological replicates. 
bioRxiv preprint doi: https://doi.org/10.1101/2022 02 14.480414. this version posted February 15,2022 . The copyright holder for this preprint (which was not certified by peer review) is the author/funder, who has granted bioRxiv a license to display the preprint in perpetuity. It is made available under aCC-BY-NC-ND 4.0 International license.

Figure 3: Mogat1 knockout prevents accumulation of glycerolipids in differentiated SVF cells.

Confluent cells were treated with tamoxifen $(4 \mathrm{nM})$ at the onset of differentiation (via DIX $\gamma$ cocktail) and harvested after 10 days. A: Depiction of lipids analyzed via LC-MS/MS B-E: Lipid content was lowered by Mogat1 knockout. Data are expressed as mean \pm S.E.M. ${ }^{*} p<0.05$ vs. vehicle, $\dagger p<0.05$ vs. control undifferentiated cells; $n=4$ biological replicates. 


\section{Figure 4: Deletion of Mogat1 attenuates the induction of triglyceride synthetic enzymes during}

adipogenesis in vitro. Confluent cells were treated with tamoxifen (4 $\mathrm{nM}$ ) at the onset of differentiation (via DIXY cocktail) and harvested after 10 days. A-D: Gene expression analysis as measured by qPCR. B: Monoacylglycerol lipase (Mgll), C: Diacylglycerol O-acyltransferase 1 and 2 (Dgat1\&2). D: Phosphatidate phosphohydrolases (Lpin1,2,\&3). Data are expressed as mean \pm S.E.M. ${ }^{*} p<0.05$ vs. vehicle, ${ }^{\dagger} p<0.05$ vs. control undifferentiated cells; $n=4$ biological replicates. 
Figure 5. Adipocyte-Specific Mogat1 knockout mice gain similar weight on a long-term high fat diet compared to littermate controls. Male adipocyte-specific Mogat1 fl/fl knockout mice (Adn-Mogat1-/-) and littermate controls (Mogat1 fl/fl) were fed a 10\% (kcal \% fat) low-fat diet (LFD) or a 60\% (kcal \% fat) high-fat diet (HFD) starting at eight weeks of age. After 16 weeks of diet, mice were fasted for 4 hours prior to sacrifice and tissue collection. A: HFD fed mice gained more weight compare to LFD fed controls and was unaffected by Mogat1 knockout. B: After 15 weeks of diet, HFD fed mice had increased fat mass compared to LFD fed mice as measured by Echo MRI. C-E: inguinal white adipose tissue (iWAT) and gonadal white adipose tissue (gWAT) but not brown adipose tissue (BAT) were increased by HFD and are expressed as \% total body weight. F, G: Samples from iWAT and gWAT were fixed and dissociated. Liberated adipocytes were measured and counted. Data are expressed as mean \pm S.E.M. ${ }^{*} p<0.05$ vs. LFD; $+p, 0.05$ vs. Mogat1 fl/fl mice; $n=5-12$ in A-E and 4-11 in F,G. 
Figure 6. Adn-Mogat1-/- mice have a similar metabolic profile as littermate controls. Male Adn-

Mogat1-/- and littermate control mice were fed a 10\% LFD or a 60\% HFD starting at eight weeks of age.

After 16 weeks of diet, mice were fasted for 4 hours prior to sacrifice and tissue collection. A-C: Plasma

triglycerides (TAG), glycerol, and free fatty acids (FFA) were measured enzymatically using commercially

available colorimetric assays. D, E: Glucose tolerance tests (GTT, $1 \mathrm{~g} / \mathrm{Kg}$ lean mass, 5 hour fast) and

insulin tolerance tests (ITT, $0.75 \mathrm{U} / \mathrm{Kg}$ lean mass, 4 hour fast) were measured at 16 weeks and 15 weeks

and were similar between groups. F: Four-hour fasted plasma insulin levels measured at sacrifice were

increased by HFD feeding. G: Liver weight as a \% total body weight was not changed by diet or genotype.

$\mathrm{H}$ : Liver triglycerides (TAG) were measured as described above and were increased by HFD feeding. Data are expressed as mean \pm S.E.M. $+p<0.05$ vs. LFD; $n=4-12$. 
Figure 7. Subcutaneous adipose tissue from adipocyte-specific Mogat1 knockout mice have a similar gene expression profile as littermate controls. Male Mogat1 $\mathrm{fl} / \mathrm{fl}$ and littermate adiponectin Cre+ (AdnMogat1-/-) mice were fed a $10 \%$ low fat diet (LFD) or a 60\% (HFD) starting at eight weeks of age. After 16 weeks of diet, mice were fasted for 4 hours prior to sacrifice and tissue collection. A schematic of genes involved in TAG metabolism. B, C: Subcutaneous iWAT adipose tissue gene expression analysis as measured by qPCR. A: Mogat1, Mogat2, Dgat1 and 2, and Lpin1. B: Peroxisome proliferator activated receptor gamma (Pparg) 1 and 2, Sterol regulatory element binding transcription factor 1 (Srebf1), Fatty acid synthase (Fasn), and Adiponectin (Adipoq) Data are expressed as mean \pm S.E.M. ${ }^{*} p<0.05$ gene effect, $+p<0.05$ diet effect; $n=5-11$. 
bioRxiv preprint doi: https://doi.org/10.1101/2022 02 14.480414 this version posted February 15,2022 . The copyright holder for this preprint

(which was not certified by peer review) is the author/funder, who has granted bioRxiv a license to display the preprint in perpetuity. It is made available under aCC-BY-NC-ND 4.0 International license.

Figure S1: Gene expression profiles of the iWAT SVF adipocyte differentiation protocol. A: Schematic of isolation and treatment protocol for the generation of SVF cell-derived adipocytes from iWAT as describe in the methods. B, C: qPCR showing classical adipocyte markers ad glycerolipid gene expression during differentiation. Data are expressed as mean \pm S.E.M. $+p<0.05$, vs Day $0 ; n=5$ biological replicates. 
bioRxiv preprint doi: https://doi.org/10.1101/2022 02 14.480414 this version posted February 15,2022 . The copyright holder for this preprint

(which was not certified by peer review) is the author/funder, who has granted bioRxiv a license to display the preprint in perpetuity. It is made available under aCC-BY-NC-ND 4.0 International license.

Figure S2: Mogat1-/- SVF cells differentiated similar to wild-type controls. A: Oil Red O staining shows similar neutral lipid accumulation in both genotypes. B: qPCR showing classical adipocyte markers ad glycerolipid gene expression during differentiation. Data are expressed as mean \pm S.E.M. ${ }^{\dagger} p<0.05$, vs Day 0; Oil Red O $n=2$ biological replicates, $\mathrm{qPCR} n=3$ biological replicates. 
bioRxiv preprint doi: https://doi.org/10.1101/2022.02 14.480414; this version posted February 15, 2022. The copyright holder for this preprint

(which was not certified by peer review) is the author/funder, who has granted bioRxiv a license to display the preprint in perpetuity. It is made available under aCC-BY-NC-ND 4.0 International license.

Figure S3: Tamoxifen treatment does not affect differentiation of wild type cells. SVF cells were

differentiated as described and treated with TAM on day indicated. After 10 days of differentiation, lipid accumulation was measured by ORO staining and quantification by extraction and measuring absorption at $492 \mathrm{~nm}$. 
Figure S4: Mogat1 knockout prevents accumulation of glycerolipids in differentiated SVF cells. Stromal vascular fractions (SVF) were isolated from iWAT of Mogat1 fl/fl mice. Confluent cells were treated with tamoxifen ( $4 \mathrm{nM}$ ) at the onset of differentiation (via DIXY cocktail) and harvested after 10 days. A, B:

DAG content was analyzed via LC-MS/MS and was lowered by Mogat1 knockout. Data are expressed as mean \pm S.E.M. ${ }^{*} p<0.05$ vs. vehicle, $+p<0.05$ vs. control undifferentiated cells; $n=4$ biological replicates. 


\section{Figure S4 continued: Mogat1 knockout prevents accumulation of glycerolipids in differentiated SVF}

cells. Stromal vascular fractions (SVF) were isolated from iWAT of Mogat1 fl/fl mice. Confluent cells

were treated with tamoxifen ( $4 \mathrm{nM}$ ) at the onset of differentiation (via DIXY cocktail) and harvested after 10 days. C-G: TAG content was analyzed via LC-MS/MS and was lowered by Mogat1 knockout. Data are expressed as mean \pm S.E.M. ${ }^{*} p<0.05$ vs. vehicle, $+p<0.05$ vs. control undifferentiated cells; $n=4$ biological replicates. 
Figure S5. Female Adipocyte-Specific Mogat1 knockout mice gain similar weight on a long-term high fat diet (HFD) compared to littermate controls. Female Mogat1 $\mathrm{fl} / \mathrm{fl}$ and littermate adiponectin Cre + (Adn-Mogat1-/-) mice were fed a 60\% HFD starting at eight weeks of age. After 16 weeks of diet, mice were fasted for 4 hours prior to sacrifice and tissue collection. A, B: Weight gain and \% lean or fat mass was unaffected by Mogat1 knockout as measured by Echo MRI. C: gWAT and iWAT but not BAT were increased by HFD and are expressed as \% total body weight. D, E: Insulin tolerance tests (ITT, $0.75 \mathrm{U} / \mathrm{Kg}$ lean mass, 4 hour fast) and glucose tolerance tests (GTT, $1 \mathrm{~g} / \mathrm{Kg}$ lean mass, 5 hour fast) were similar between groups. Data are expressed as mean \pm S.E.M. $n=7-9 ; n=3-4$ for ECHO MRI. 


\section{Figure S6. Bulk RNA sequencing analysis of iWAT shows few differences between Mogat1-/- and}

littermate controls on a HFD. A: Heatmap of merged differential expression data from iWAT from

Mogat1-/- littermate controls on a HFD. B: Volcano plots of merged differential expression data was

graphed as $\log _{2}$ fold change verses $-\log _{10}$ unadjusted $p$ value. C: List of significantly changed genes. Gene

expression changes were considered meaningful if they were $>\log _{2}$ fold change and had an adjusted $p$

value of $<0.05 \mathrm{vs}$. $\mathrm{fl} / \mathrm{fl}$ mice on HFD. 
bioRxiv preprint doi: https://doi.org/10.1101/2022.02 14.480414; this version posted February 15, 2022. The copyright holder for this preprint

(which was not certified by peer review) is the author/funder, who has granted bioRxiv a license to display the preprint in perpetuity. It is made available under aCC-BY-NC-ND 4.0 International license.

Figure S7: Mogat1 ablation in adipocytes alters metabolic pathways. A-D: Graphical representation of pathway analysis showing top 10 significant signal direction changes of GO biological process and KEGG Signaling and Metabolism pathways. The color of the pathway label indicates the $p$ value and the $x$-axis is the mean log fold change. 
Table S1. Primer sequences used for RT-qPCR

\begin{tabular}{|c|c|c|c|}
\hline Gene & Species & Forward 3'-5' & Reverse 3'-5' \\
\hline Adiponectin & mouse & tgttcctcttaatcctgccca & ccaacctgcacaagttccctt \\
\hline Dgat1 & mouse & tgcagtttggagaccgcgagtt & cacccatttgctgctgccatgt \\
\hline Dgat2 & mouse & ggtgatctttgaggagggttc & gtacaggtcgatgtctttctgg \\
\hline Fasn & mouse & gtctggaaagctgaaggatctc & tgcctctgaaccactcacac \\
\hline Lpin1 & mouse & ccctcgatttcaacgcacct & gcagcctgtggcaattca \\
\hline Lpin2 & mouse & gaagtggcggctctctatttc & agagggttacatcaggcaagt \\
\hline Lpin3 & mouse & tcacccttccacgtgcgcttc & tcttcctcatcactgtccagctcct \\
\hline Mgll & mouse & cggacttccaagtttttgtcaga & gcagccactaggatggagatg \\
\hline Mogat1 (exon 4) & mouse & tgtcttgtcaaaagcgacgat & acaacgggaaacagaaccaga \\
\hline Mogat1 (full length) & mouse & tggtgccagtttggttccag & tgctctgaggtcgggttca \\
\hline Mogat2 & mouse & tgggagcgcaggttacaga & caggtggcatacaggacaga \\
\hline Pparg1 & mouse & ggaagaccactcgcattcctt & gtaatcagcaaccattgggtca \\
\hline Pparg2 & mouse & tcgctgatgcactgcctatg & gagaggtccacagagctgatt \\
\hline Srebf1 & mouse & ggcactaagtgccctcaacct & gccacatagatctctgccagtgt \\
\hline
\end{tabular}




\section{Figure 1}

A

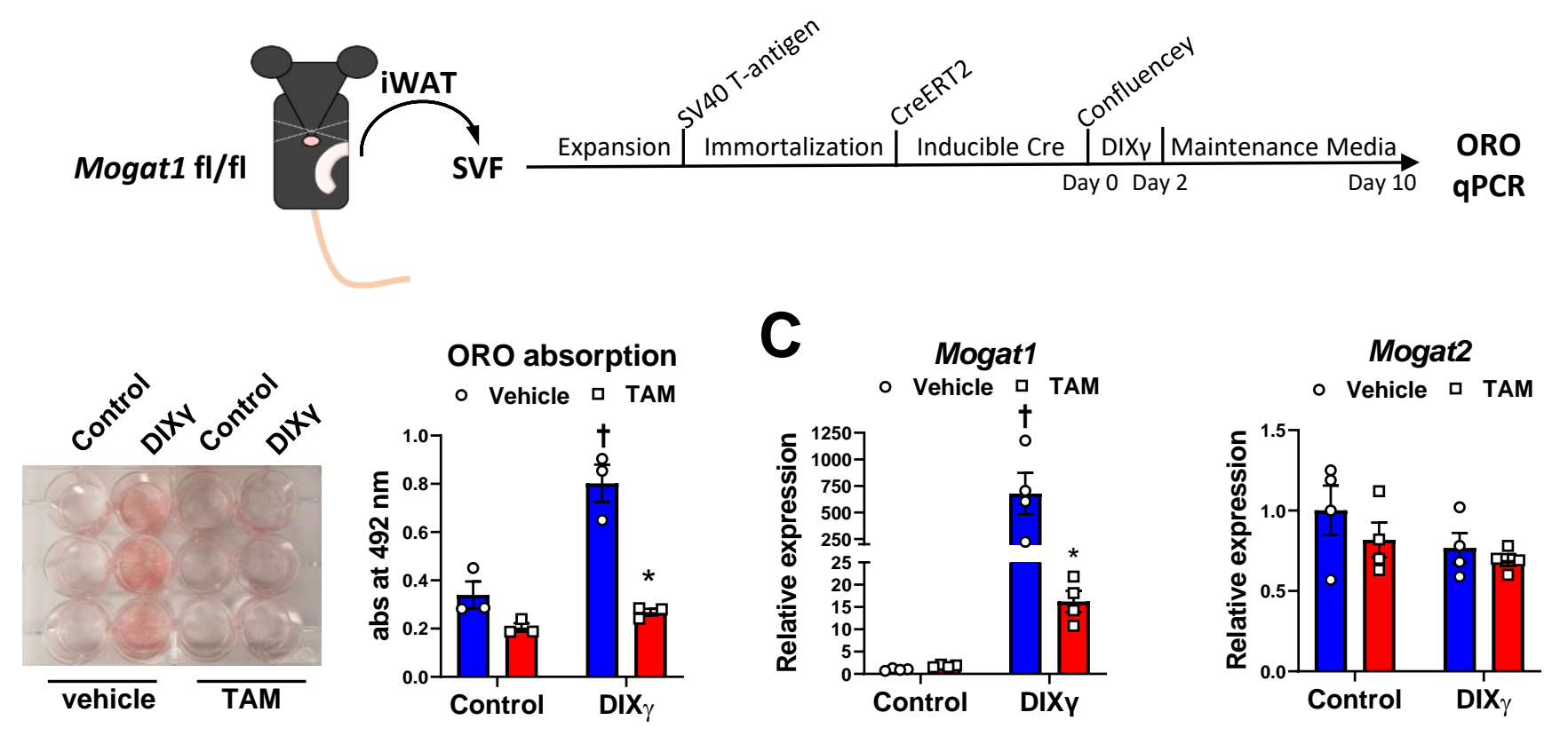

Figure 1: Mogat1 expression is required for adipocyte differentiation initiation in vitro. Stromal vascular fractions (SVF) were isolated from iWAT adipose tissue of Mogat1 $\mathrm{fl} / \mathrm{fl}$ mice. Cells were immortalized with SV40 T antigen prior to infection with an inducible CreERT2. A: Diagram of treatment protocol for the generation of SVF and inducible Mogat1 cell lines as described in the methods B, C: Cre recombinase activity to delete Mogat1 was induced with the addition 4 nM tamoxifen on Day 0 while other cells were treated with vehicle. B: After 10 days of differentiation, lipid accumulation was reduced by Mogat1 knockout as shown by a representative ORO staining and quantification by extraction and measuring absorption at $492 \mathrm{~nm}$. C: Gene expression analysis as measured by qPCR. Mogat1 gene expression is induced by differentiation and inhibited by TAM while Mogat2 was unaffected by differentiation or TAM treatment. Data are expressed as mean \pm S.E.M. ${ }^{*} p<0.05$, comparisons are indicated; $n=3-4$ biological replicates. 


\section{Figure 2}

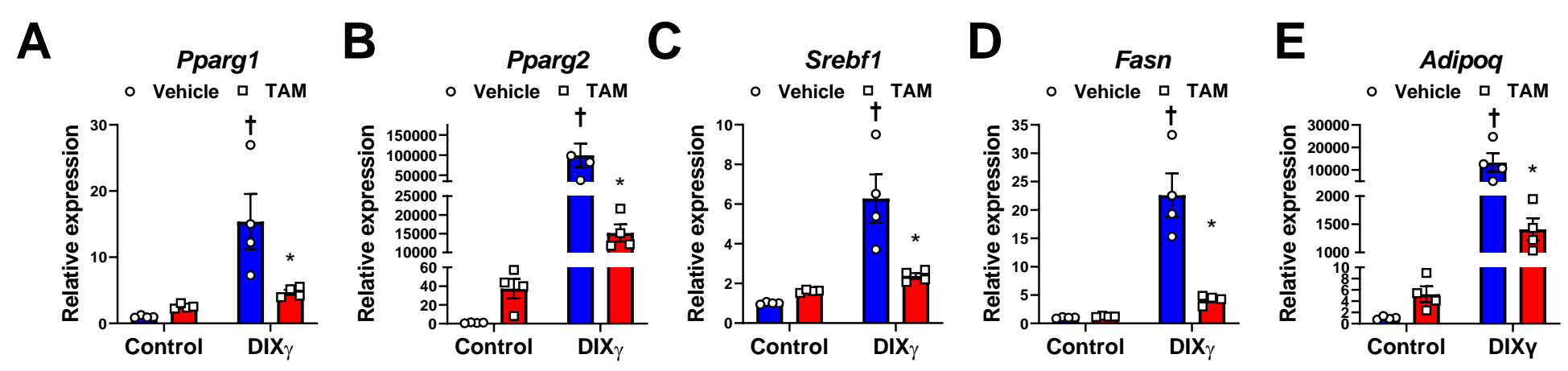

Figure 2: Mogat1 is required for adipogenic gene expression in vitro. Confluent cells were treated with tamoxifen (4 nM) at the onset of differentiation (via DIXY cocktail) and harvested after 10 days. A-E: Gene expression analysis as measured by qPCR: Peroxisome proliferator activated receptor gamma (Pparg) 1 and 2, Sterol regulatory element binding transcription factor 1 (Srebf1), Fatty acid synthase (Fasn), and Adiponectin (Adipoq) were increased during differentiation and reduced by Mogat1 knockout. Data are expressed as mean \pm S.E.M. ${ }^{*} p<0.05$ vs. vehicle, $+p<0.05$ vs. control undifferentiated cells; $n=$ 4 biological replicates. 
A

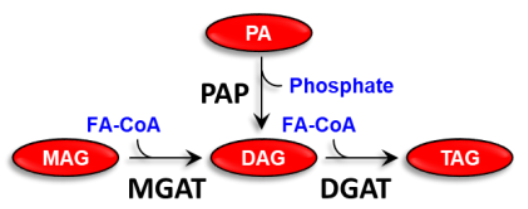

C : Control $\begin{gathered}\text { Control+TAM } \\ \text { DIX }\end{gathered}$

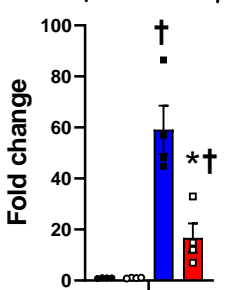<smiles></smiles>

B

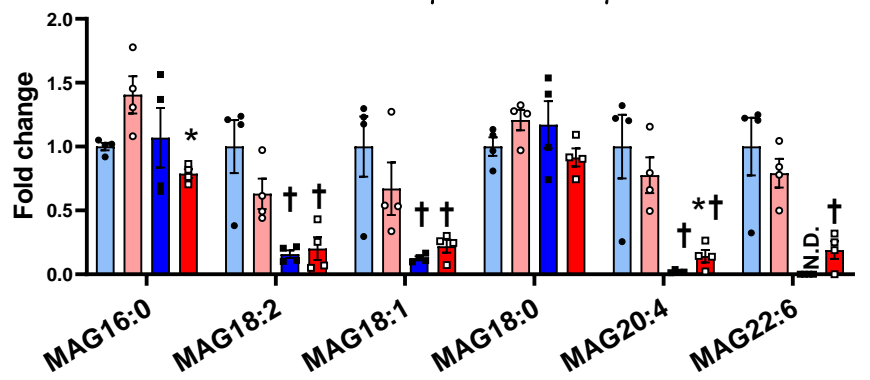

\section{MAG}

: Control : ControltTAM
D

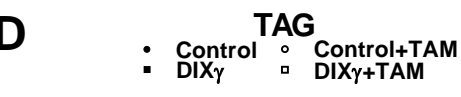

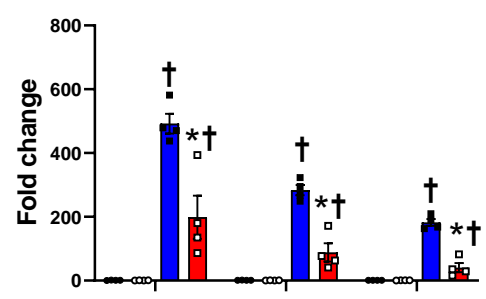

TAG

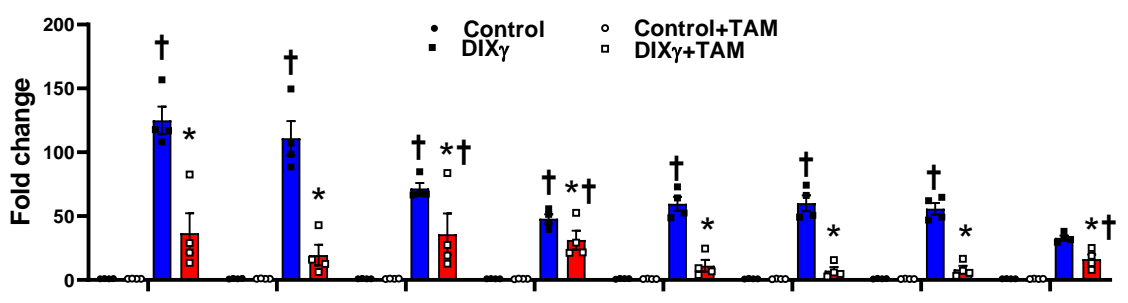

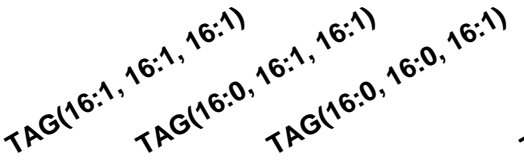

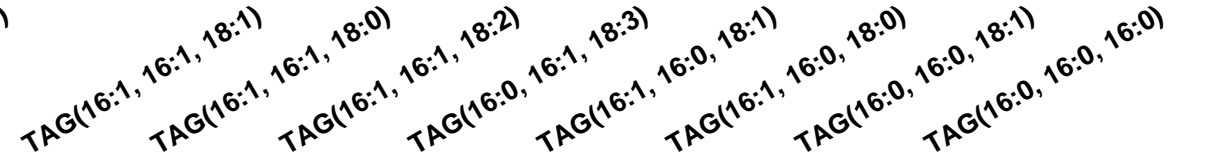

E

PA

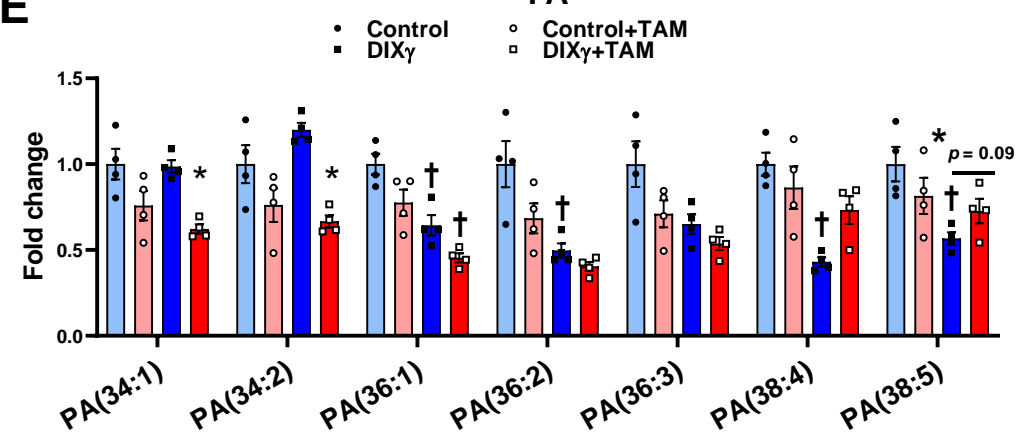

Figure 3: Mogat1 knockout prevents accumulation of glycerolipids in differentiated SVF cells. Confluent cells were treated with tamoxifen $(4 \mathrm{nM})$ at the onset of differentiation (via DIXY cocktail) and harvested after 10 days. A: Depiction of lipids analyzed via LC-MS/MS B-E: Lipid content was lowered by Mogat1 knockout. Data are expressed as mean \pm S.E.M. ${ }^{*} p<0.05$ vs. vehicle, $+p<0.05$ vs. control undifferentiated cells; $n=4$ biological replicates. 


\section{Figure 4}

A

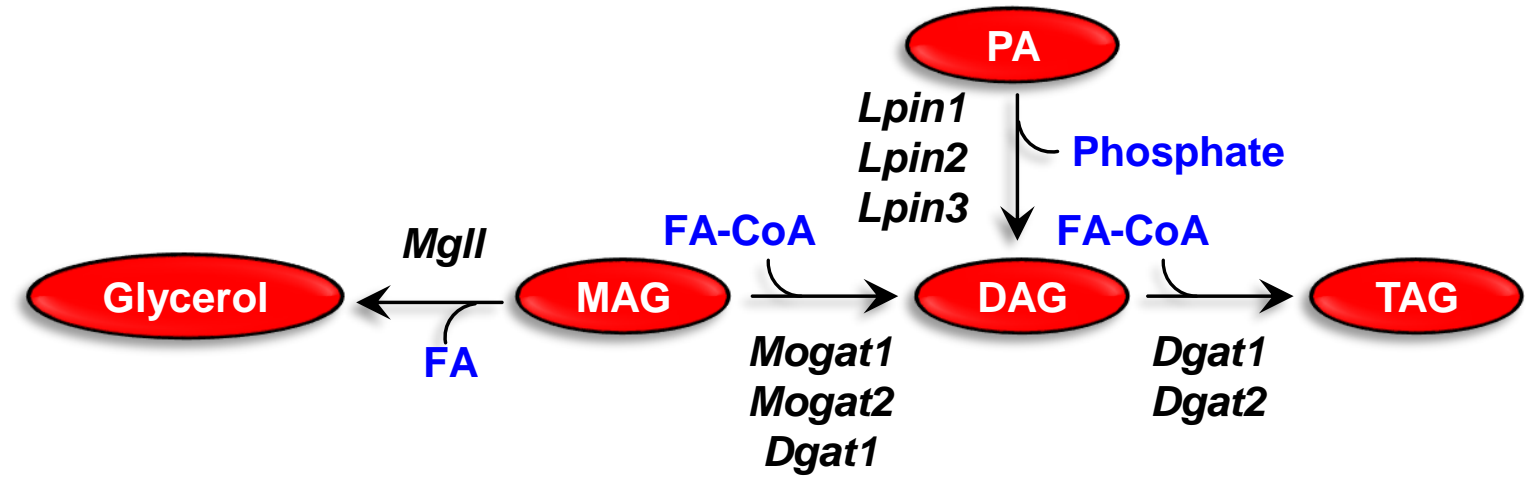

B

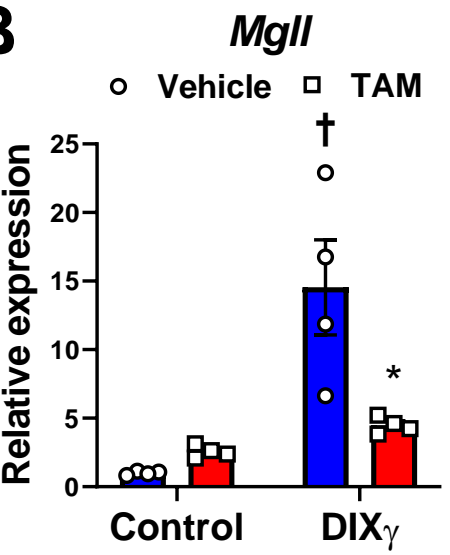

D
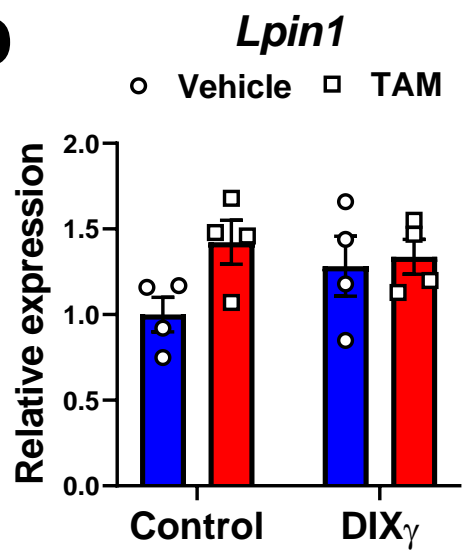

C - Vehicle 口 TAM

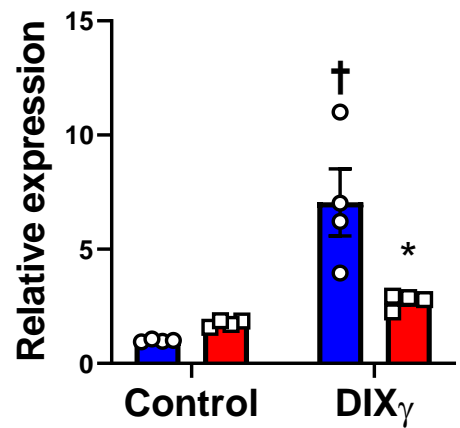

Lpin2

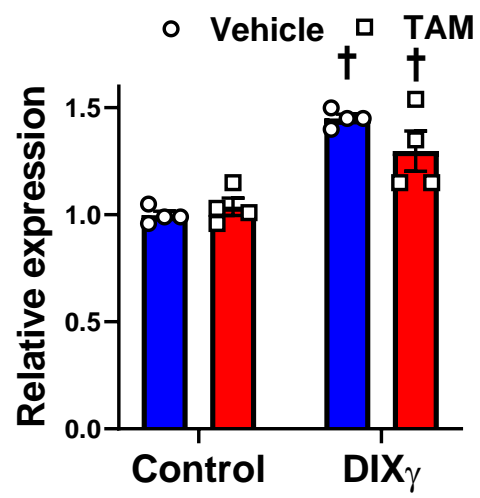

Dgat2

- Vehicle $\square$ TAM

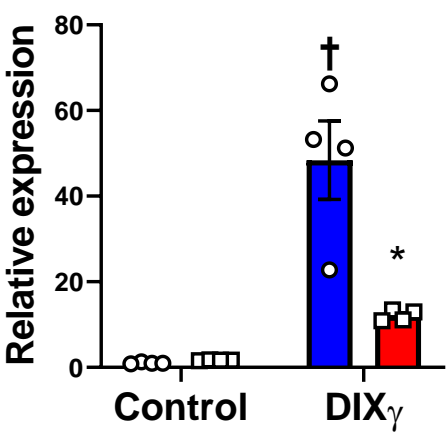

Lpin3

○ Vehicle $\square$ TAM

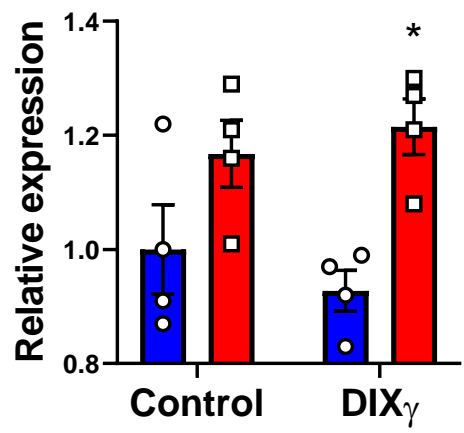

Figure 4: Deletion of Mogat1 attenuates the induction of triglyceride synthetic enzymes during adipogenesis in vitro. Confluent cells were treated with tamoxifen $(4 \mathrm{nM})$ at the onset of differentiation (via DIXY cocktail) and harvested after 10 days. A: Schematic of glycerolipid synthesis genes. B-D: Gene expression analysis as measured by qPCR. B: Monoacylglycerol lipase (Mgll), C: Diacylglycerol O-acyltransferase 1 and 2 (Dgat1\&2). D: Phosphatidate phosphohydrolases (Lpin1,2,\&3). Data are expressed as mean \pm S.E.M. $* p<0.05$ vs. vehicle, $+p<0.05$ vs. control undifferentiated cells; $n=4$ biological replicates. 


\section{A}

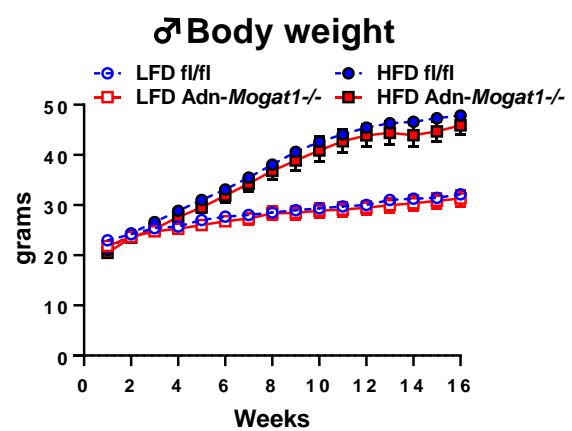

B

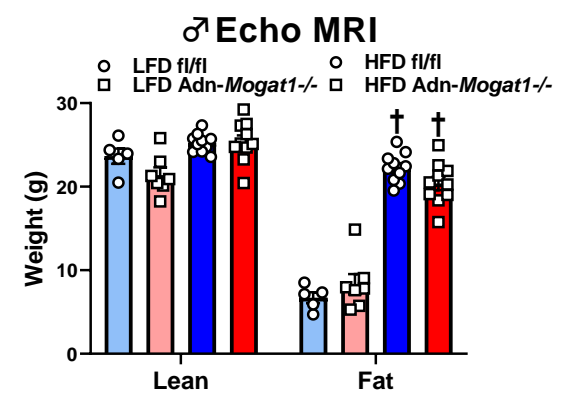

C

iWAT
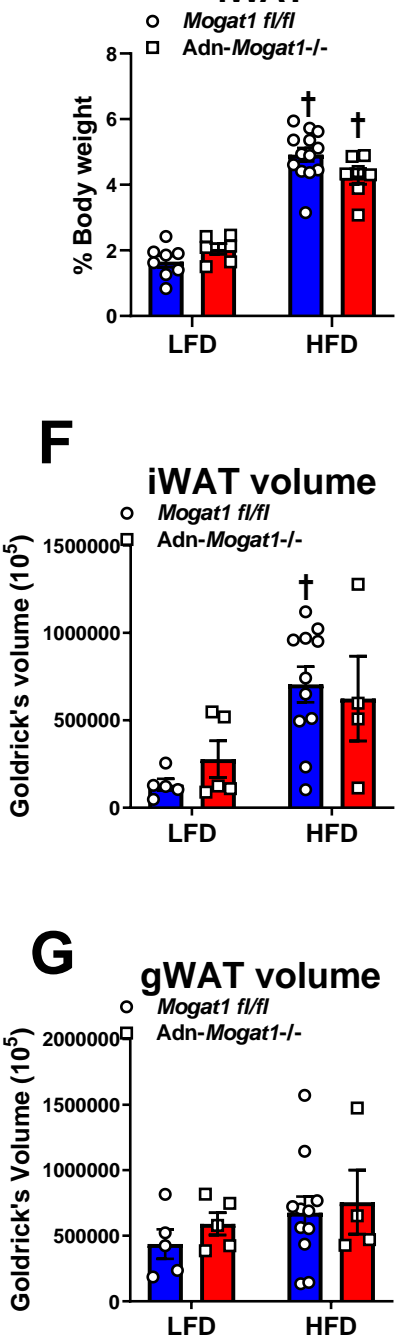

D
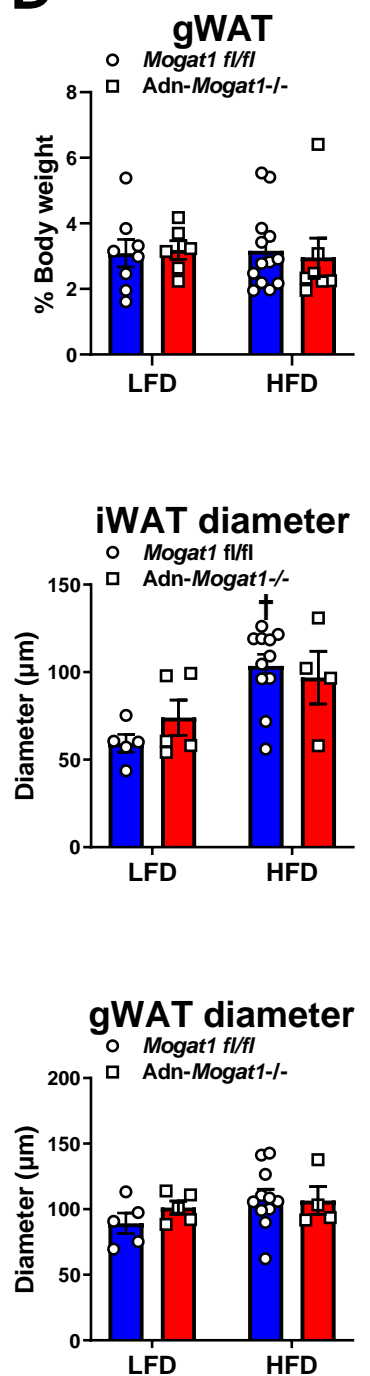

$\mathbf{E}$
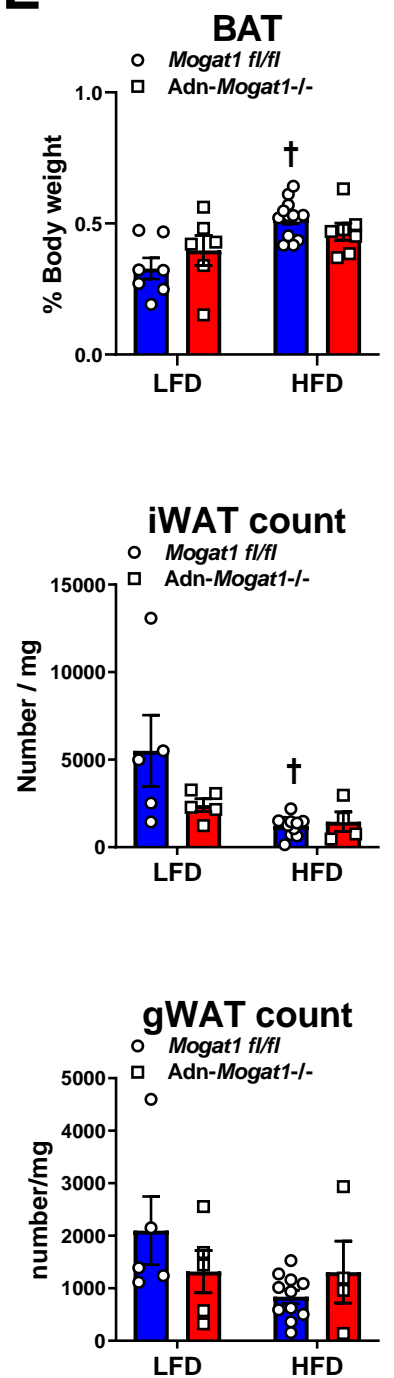

Figure 5. Adipocyte-Specific Mogat1 knockout mice gain similar weight on a long-term high fat diet compared to littermate controls. Male adipocyte-specific Mogat1 fl/fl knockout mice (Adn-Mogat1-/-) and littermate controls (Mogat1 fl/fl) were fed a $10 \%$ (kcal \% fat) low-fat diet (LFD) or a $60 \%$ (kcal \% fat) high-fat diet (HFD) starting at eight weeks of age. After 16 weeks of diet, mice were fasted for 4 hours prior to sacrifice and tissue collection. A: HFD fed mice gained more weight compare to LFD fed controls and was unaffected by Mogat1 knockout. B: After 15 weeks of diet, HFD fed mice had increased fat mass compared to LFD fed mice as measured by Echo MRI. C-E: inguinal white adipose tissue (iWAT) and gonadal white adipose tissue (gWAT) but not brown adipose tissue (BAT) were increased by HFD and are expressed as \% total body weight. F, G: Samples from iWAT and gWAT were fixed and dissociated. Liberated adipocytes were measured and counted. Data are expressed as mean \pm S.E.M. ${ }^{*} p<0.05$ vs. LFD; ${ }^{\dagger} p, 0.05$ vs. Mogat1 $\mathrm{fl} / \mathrm{fl}$ mice; $n=5-12$ in A-E and 4-11 in F,G. 

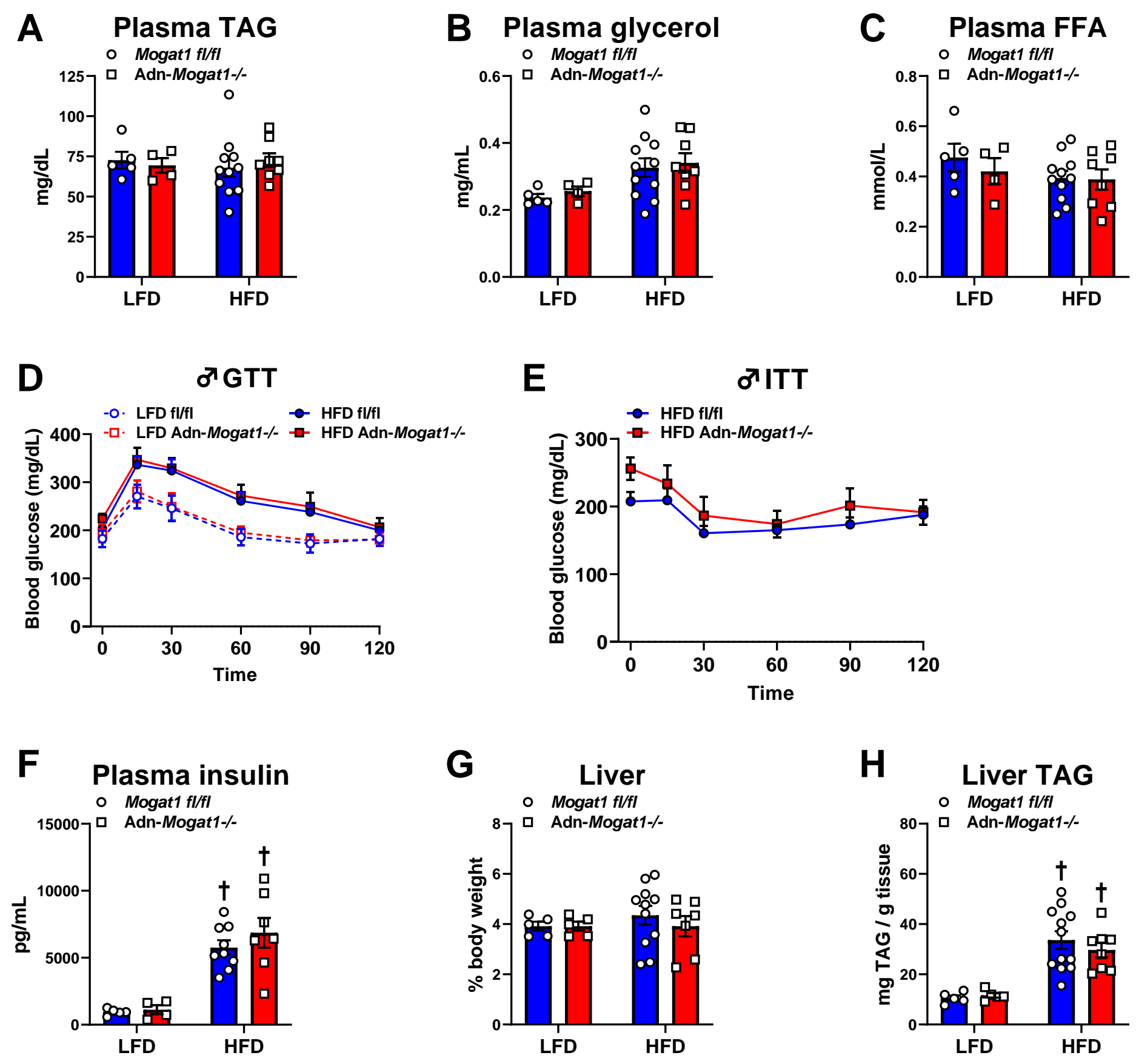

Figure 6. Adn-Mogat1-/- mice have a similar metabolic profile as littermate controls. Male Adn-Mogat1-/- and littermate control mice were fed a 10\% LFD or a 60\% HFD starting at eight weeks of age. After 16 weeks of diet, mice were fasted for 4 hours prior to sacrifice and tissue collection. A-C: Plasma triglycerides (TAG), glycerol, and free fatty acids (FFA) were measured enzymatically using commercially available colorimetric assays. D, E: Glucose tolerance tests (GTT, $1 \mathrm{~g} / \mathrm{Kg}$ lean mass, 5 hour fast) and insulin tolerance tests (ITT, $0.75 \mathrm{U} / \mathrm{Kg}$ lean mass, 4 hour fast) were measured at 16 weeks and 15 weeks and were similar between groups. F: Four-hour fasted plasma insulin levels measured at sacrifice were increased by HFD feeding. G: Liver weight as a \% total body weight was not changed by diet or genotype. H: Liver triglycerides (TAG) were measured as described above and were increased by HFD feeding. Data are expressed as mean \pm S.E.M. $+p<0.05$ vs. LFD; $n=4-12$. 


\section{Figure 7}

A

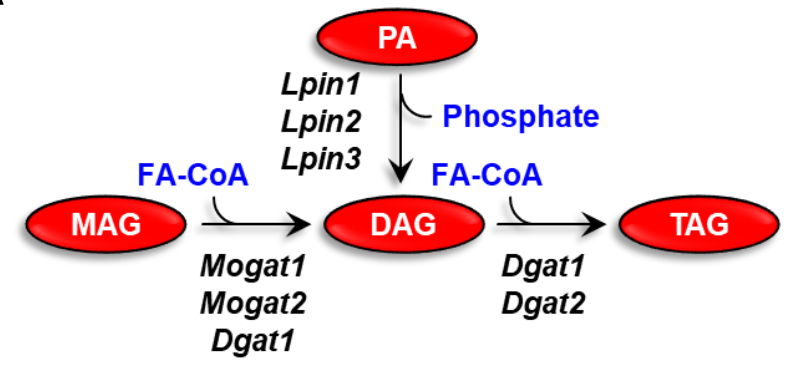

B
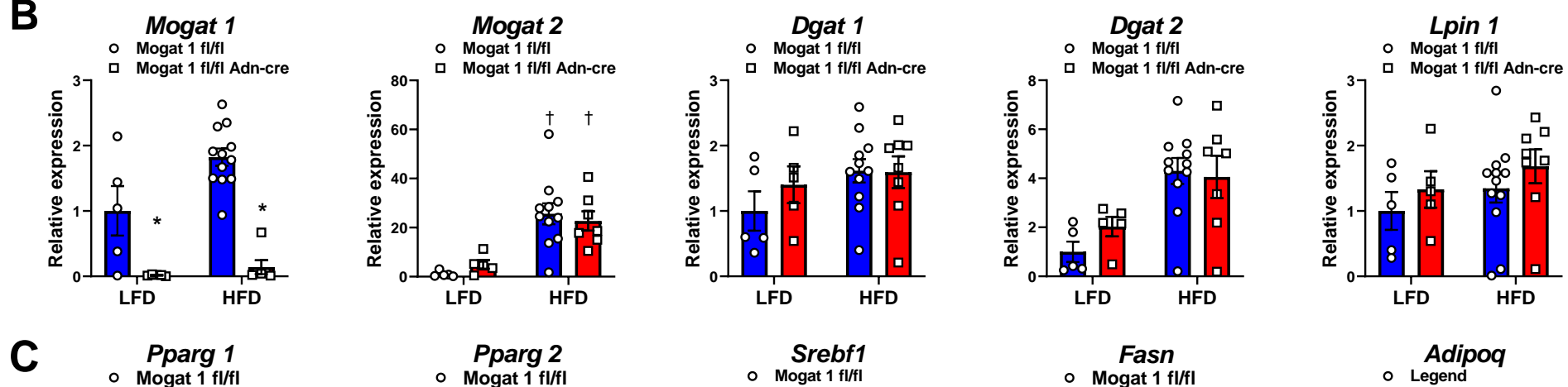

Pparg 2
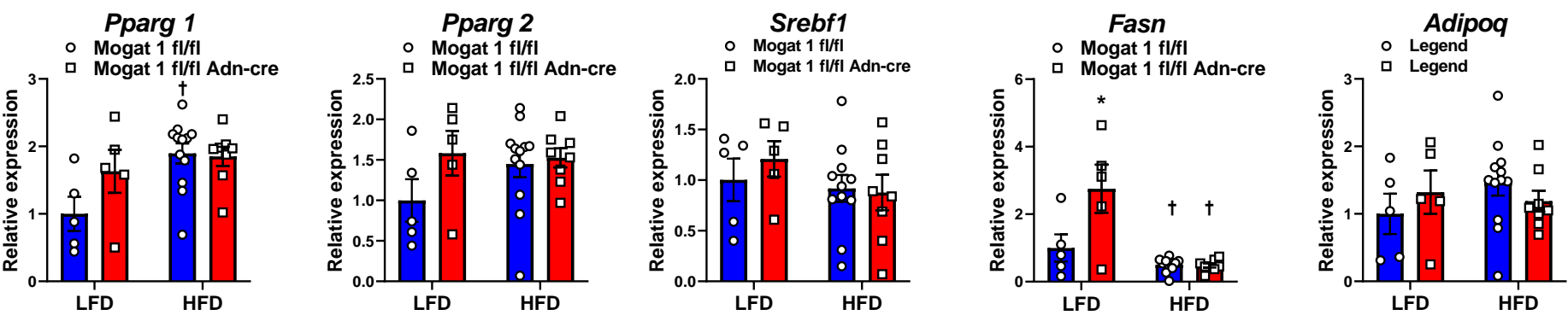

Figure 7. Subcutaneous adipose tissue from adipocyte-specific Mogat1 knockout mice have a similar gene expression profile as littermate controls. Male Mogat1 fl/fl and littermate adiponectin Cre+ (Adn-Mogat1-/-) mice were fed a 10\% low fat diet (LFD) or a 60\% (HFD) starting at eight weeks of age. After 16 weeks of diet, mice were fasted for 4 hours prior to sacrifice and tissue collection. A schematic of genes involved in TAG metabolism. B, C: Subcutaneous iWAT adipose tissue gene expression analysis as measured by qPCR. A: Mogat1, Mogat2, Dgat1 and 2, and Lpin1. B: Peroxisome proliferator activated receptor gamma (Pparg) 1 and 2, Sterol regulatory element binding transcription factor 1 (Srebf1), Fatty acid synthase (Fasn), and Adiponectin (Adipoq) Data are expressed as mean \pm S.E.M. ${ }^{*} p<0.05$ gene effect, $+p<0.05$ diet effect; $n$ $=5-11$. 